\title{
Les armes miraculeuses
}

\section{En mémoire de Sarah Maldoror}

\author{
Alice Leroy et Raquel Schefer
}

Citer cet article : Leroy Alice, Schefer Raquel (2021), « Les armes miraculeuses. En mémoire de Sarah

Maldoror », Revue d'Histoire Contemporaine de l'Afrique, $\mathrm{n}^{\circ} 1,167-177$, en ligne. URL :

https://oap.unige.ch/journals/rhca/article/view/01.leroy_schefer

Mise en ligne : 7 janvier 2021

DOI : https://doi.org/10.51185/journals/rhca.2021.e296

Et surtout mon corps aussi bien que mon âme, gardez-vous de vous croiser les bras en l'attitude stérile du spectateur, car la vie n'est pas un spectacle, car une mer de douleurs n'est pas un proscenium, car un homme qui crie n'est pas un ours qui danse... ${ }^{1}$

Née en 1928, Sarah Maldoror s'était donné un nom à la mesure de ses engagements artistiques et politiques. Auprès de ses amis de Présence Africaine ou des militants du Front de Libération Nationale de l'Algérie (FLN) et du Parti Africain pour l'indépendance de la GuinéeBissau et du Cap-Vert (PAIGC), elle accompagnait les mouvements de décolonisation et les luttes de libération africaine. Réalisatrice de près de quarante films de tous formats, elle reste pourtant méconnue. Elle s'est éteinte le 13 avril 2020 des suites de la Covid-19.

1 Césaire Aimé (2000 [1947]), Cahier d'un retour au pays natal, Paris, Présence africaine. 


\section{Image $n^{\circ} 1:$ Portrait de Sarah Maldoror ${ }^{2}$}

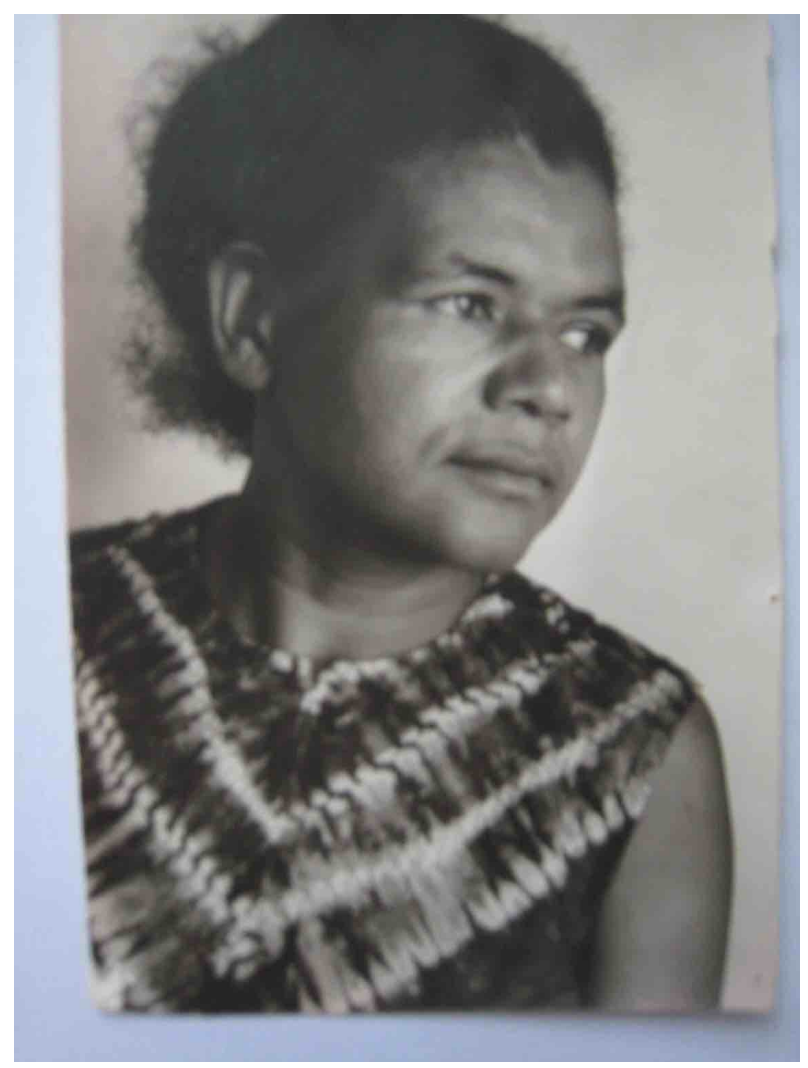

\section{Sortir de l'ombre}

Les films de Sarah Maldoror ont longtemps fait partie de ce que Roberto Moura appelle le " cinéma invisible ${ }^{3}$ »:

[Un cinéma] qui n'a pas été incorporé par l'histoire et qui est donc resté oublié, mais qui existe toujours et s'efforce d'être vu. Ce sont des films qui ne sont pas encore devenus définitivement du "cinéma invisibilisé" [sid] ; des films dont on s'est désintéressé, favorisant la détérioration des copies; des films qui peuvent encore être mentionnés par certains documents [...] mais qui malheureusement ne peuvent pas être vus ${ }^{4}$.

La filmographie de la cinéaste s'inscrit aujourd'hui encore dans les zones d'ombre d'une histoire non canonique du cinéma, histoire qu'il importe de restituer et de resignifier au regard d'un contre-récit féminin et féministe sur les cinémas révolutionnaires, incluant notamment la figure d'Esfir Choub en Union Soviétique, ou encore celles de Sara Gómez à Cuba et de Josefina Crato en Guinée-Bissau. Cependant, le travail de conservation, de restauration et de numérisation des films de Sarah Maldoror impulsé par ses filles Annouchka de Andrade et Henda Ducados au cours des dix dernières années a contribué de façon décisive à la redécouverte de son œuvre: I'Arsenal de Berlin a entrepris la restauration de Monagambée

\footnotetext{
2 Toute les images proviennent des archives d'Annouchka de Andrade. Courtoisie d'Annouchka de Andrade.

3 Moura Roberto (2002), "Le renouveau du dialogue cinématographique France-Brésil, et la contre représentation de la société brésilienne sous les gouvernements militaires dans le "cinéma invisible" des années 1969-90", Histoire et sociétés de l'Amérique Latine, 2002, 14, pp. 11-32.

4 Ibid., pp. 20-21.
} 
(1969) tandis que le Musée Reina Sofía à Madrid dédiait une rétrospective à la réalisatrice en 2019. D'autres rétrospectives sont en cours de préparation à Paris et ailleurs.

\section{Paris, Moscou, Alger}

Images $n^{\circ} 2$ : Photo de tournage de La Bataille d'Alger (Gillo Pontecorvo, 1966)

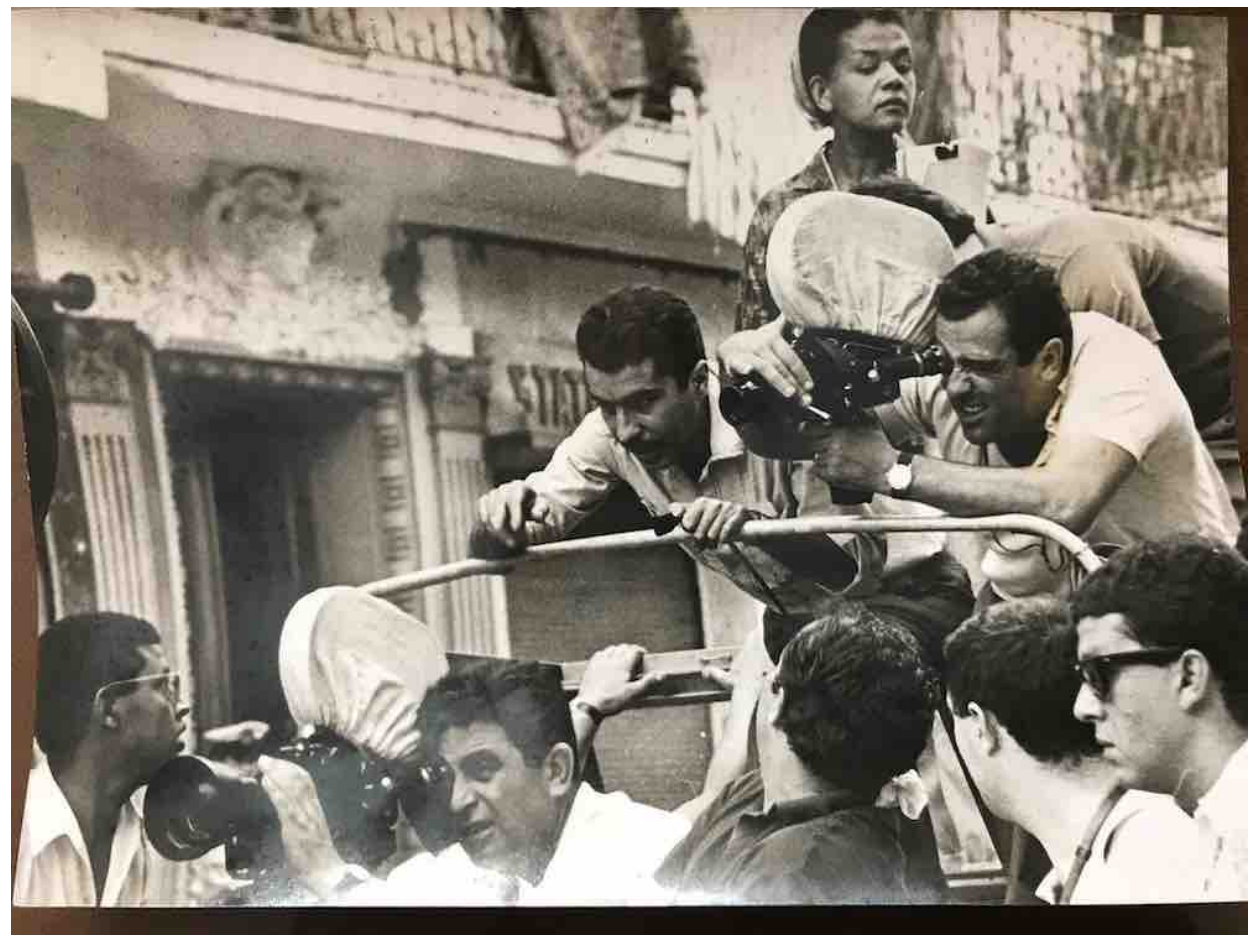

Image $\mathrm{n}^{\circ} 3$ : Sarah Maldoror au travail sur La Bataille d'Alger

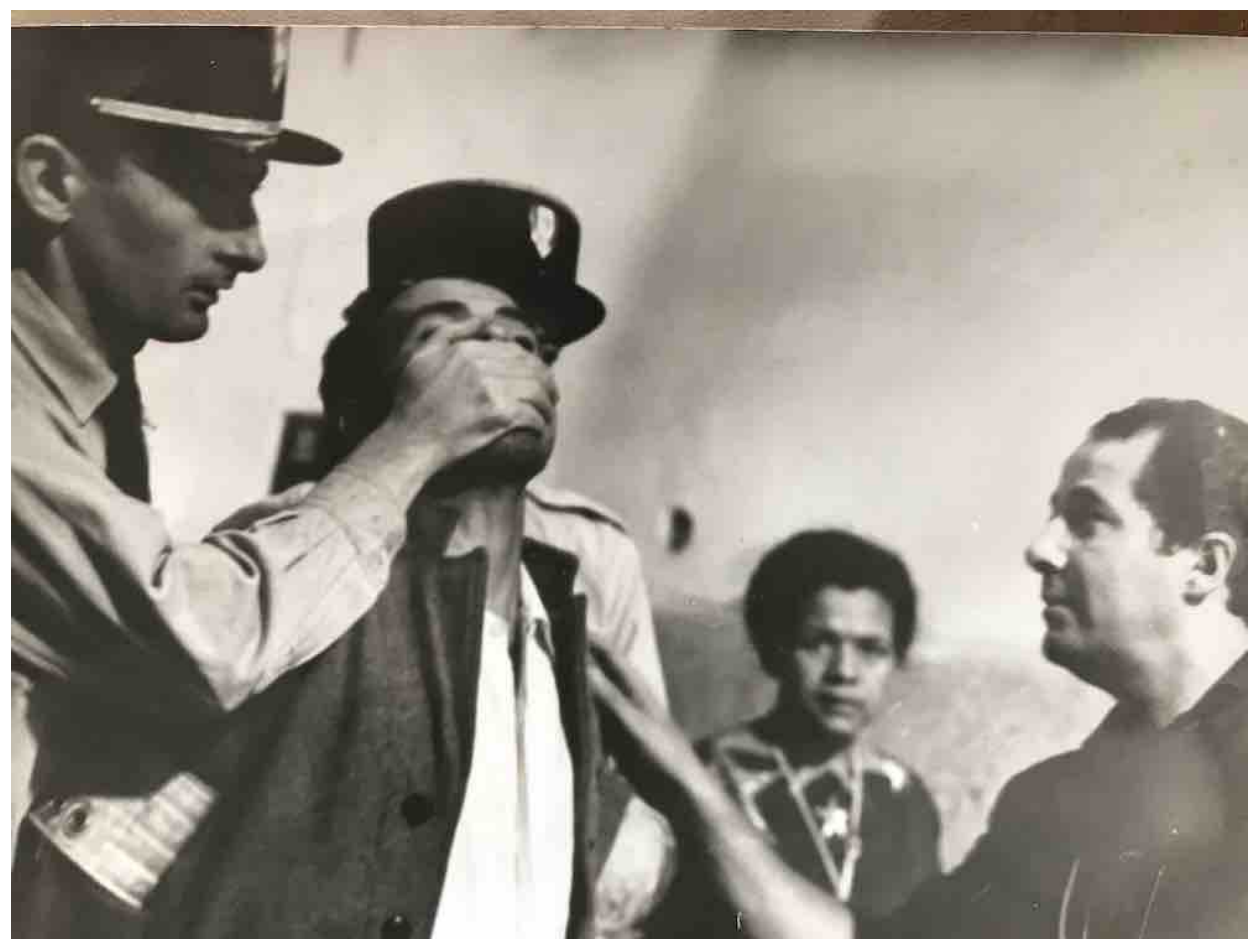


Née d'une mère gersoise et d'un père guadeloupéen, Sarah Maldoror arrive à Paris au début des années 1950. C'est ici qu'au contact de la librairie Présence africaine, elle fonde des amitiés durables avec Léopold Sédar Senghor, Édouard Glissant, Alioune Diop et Aimé Césaire, auquel elle dédiera plusieurs films et portraits. Elle se choisit un patronyme à la mesure de sa personnalité : Maldoror, un nom comme un poème et une promesse qui la jette dans les arts 5 . Le théâtre d'abord, avec la compagnie Les Griots fondée avec trois amis étudiants. Ensemble, ils montent les pièces de Jean Genêt et celles de Jean-Paul Sartre, qui paie lui-même ses droits d'auteurs à Gallimard pour la mise en scène de sa pièce Huis-clos. Le cinéma ensuite, parce que parmi ces écrivains, poètes et metteurs en scène, il n'y a pas de cinéaste. Chris Marker, un autre de ses amis, lui conseille d'aller apprendre le cinéma en Union Soviétique. Elle le prend au mot et part pour Moscou en 1961 étudier auprès de Marc Donskoï. Deux ans plus tard, elle est à Rabat, au Maroc, où elle rejoint son compagnon Mário Pinto de Andrade, qu'elle a rencontré en 1956 à Paris lors du premier congrès des écrivains et artistes noirs. À Rabat d'abord, Alger ensuite, les leaders révolutionnaires des mouvements de libération africains organisent la lutte. Alger est alors la Capitale des révolutionnaires, ainsi que le résume bien le titre du film de Gordian Troeller et Marie-Claude Deffarge en 1972. Le Festival Panafricain d'Alger de 1969, auquel participent des délégations du Mouvement Populaire de Libération de I'Angola (MPLA), ainsi que du Parti Africain pour l'indépendance de la Guinée-Bissau et du CapVert (PAIGC) et du Front de Libération du Mozambique (FRELIMO), contribue à renforcer les connexions internationalistes des mouvements de libération. Cette rencontre témoigne de l'effervescence révolutionnaire de la capitale algérienne dans les années 1960. De Frantz Fanon aux Black Panthers, de l'Organisation de Libération de la Palestine (OLP) au Vietcong, sans oublier les exilés politiques portugais, espagnols et brésiliens, tous les révolutionnaires et les mouvements de libération passent par Alger. Le Festival Panafricain démontre le rôle de la culture dans les luttes de libération africaines, ce que l'intertitre final du film documentaire de William Klein souligne : "La culture africaine sera révolutionnaire ou ne sera pas » (Festival Panafricain d'Alger 1969, 1969).

C'est à Alger que la carrière cinématographique de Sarah Maldoror débute. En 1966, elle travaille d'abord aux côtés de Ahmed Lallem sur le tournage de son moyen-métrage Elles, avec des lycéennes algériennes qui témoignent de leurs vies et de leurs rêves au lendemain de I'indépendance du pays. La même année, elle est assistante de Gillo Pontecorvo dans La bataille d'Alger et responsable du casting de figuration des scènes tournées dans la Casbah. Chargée en particulier de recruter des femmes, Sarah Maldoror va mettre à profit ces deux expériences pour instaurer des figures féminines au cœur de ce qu'elle appelle son « cinéma de résistance ». À la différence de Pontecorvo, le cinéma de Sarah Maldoror raconte les luttes de libération à travers le parcours de personnages féminins qui incarnent les formes émancipatrices d'une indiscipline poétique et politique.

\footnotetext{
${ }^{5}$ Dans un entretien que Sarah Maldoror avait accordé à Alice Leroy, elle expliquait avoir été marquée par le livre du Compte de Lautréamont, Les Chants de Maldoror. Mais plus qu'une référence à la personnalité du personnage, c'est surtout pour sa sonorité qu'elle avait choisi ce pseudonyme.
} 


\section{Monamgambée et Sambizanga, naissance d'une cinéaste}

Image $n^{\circ} 4$ : Photogramme de Sambizanga (1973). La prison de la PIDE, la police politique du régime fasciste-colonial portugais

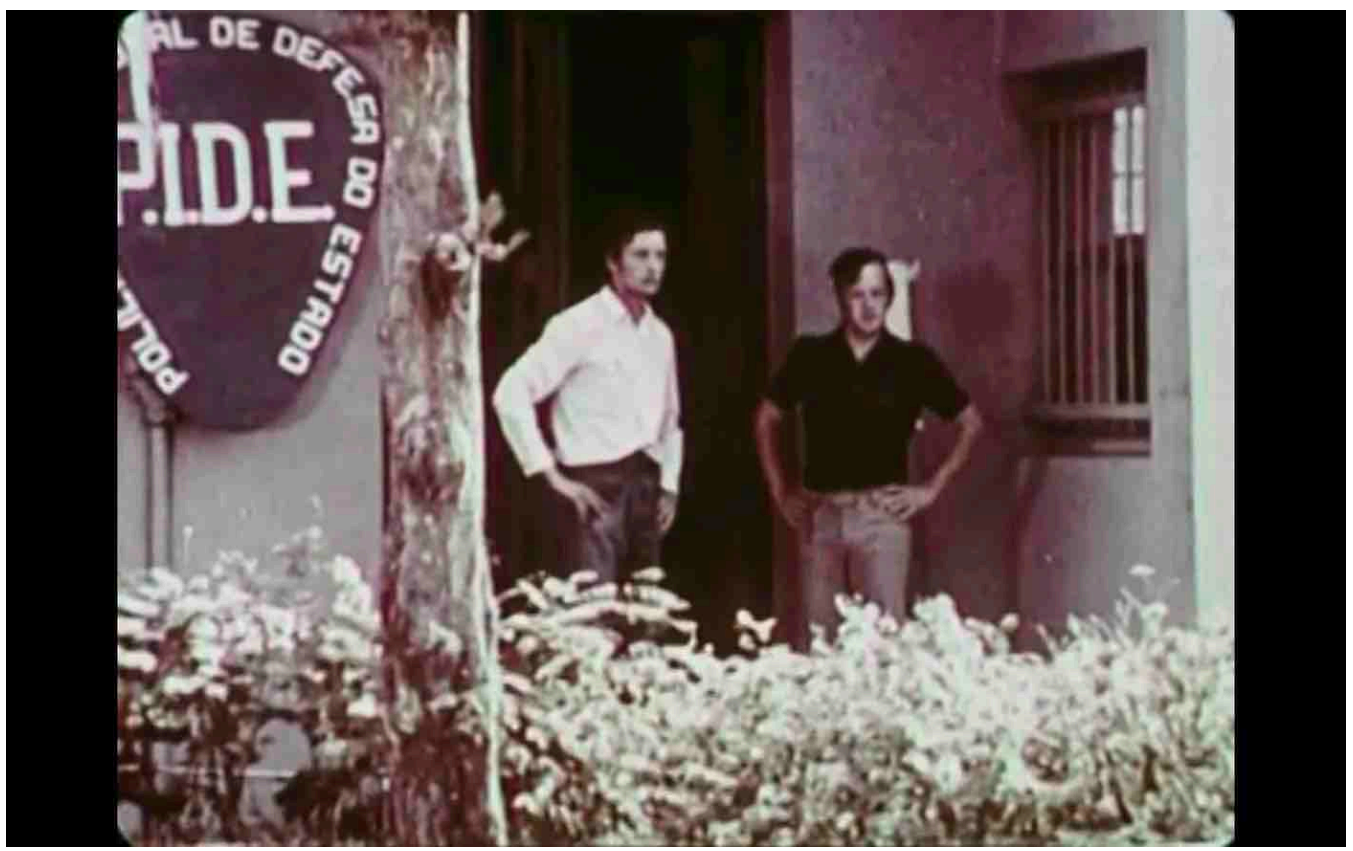

À Alger, Maldoror s'est liée avec les militants du MPLA, en particulier Elisa et Carlos Pestana qui incarnent les deux personnages principaux de son premier court métrage, Monamgambée $(1969)^{6}$. Produit par le FLN algérien et par le MPLA, le film est tourné en trois semaines près d'Alger, dans une forme d'urgence politique, tandis que la lutte se poursuit. Ce récit de torture, adapté d'une nouvelle de l'écrivain angolais Luandino Vieira (Le Complet de Mateus, 1962), décrit le sort réservé aux prisonniers politiques du régime de Salazar en Afrique lusophone dans les prisons coloniales. Maldoror y filme les gestes d'amour comme des actes de résistance et la résilience des corps noirs comme leur dernière arme. Réalisé quatre ans plus tard, Sambizanga (1973), son premier long métrage accessible aujourd'hui, est une autre adaptation de Luandino Vieira (La Vraie vie de Domingos Xavier, 1961). Ici encore, la cinéaste oppose la violence coloniale à la puissance des liens qui unissent ceux qui s'aiment et luttent ensemble. L'invisibilité - aujourd'hui, relative - de l'œuvre de Maldoror s'explique en partie par sa singulière articulation entre les formes du politique et une politique des formes à travers l'exercice d'une fabulation narrative et esthétique contraire aux normes souvent rigides du cinéma militant. Mais cette invisibilité ne peut pas non plus être séparée des facteurs qui tiennent au lieu et à la position d'énonciation - tout particulièrement, à la question de genre, aussi bien qu'à la complexe histoire du processus de libération anticoloniale des pays africains de langue portugaise.

En mai 1974, un an avant l'indépendance, le compagnon de Maldoror, I'homme de lettres, idéologue et premier président du MPLA Mário Pinto de Andrade, fonde avec son frère Joaquim Pinto de Andrade la faction « Revolta Activa » (Révolte active) au sein du mouvement de libération. Inquiète de la dérive autoritaire du MPLA autour de la figure d'Agostinho Neto,

\footnotetext{
6 Elisa Pestana est aussi la protagoniste de Sambizanga (1973).
} 
futur premier président de l'Angola, Pinto de Andrade rompt avec le mouvement qu'il avait cofondé en 1956 selon l'histoire officielle angolaise ${ }^{7}$. Forcé de s'exiler, il devient coordinateurgénéral du Conseil national de la culture (1976-1978), puis ministre de I'Information et de la Culture de la Guinée-Bissau (1978-1980, jusqu'au coup d'État de novembre de cette année). Le devenir de Monamgambée et de Sambizanga - pourtant considéré comme le premier long métrage de fiction en Angola - est scellé. Pinto de Andrade devenu persona non grata dans son pays natal, les deux films de sa compagne sont voués à l'invisibilité. En 2020, la cinéaste reçoit finalement, à titre posthume, le Prix national de la culture et des arts de l'Université catholique d'Angola.

C'est en Guinée-Bissau et au Cap-Vert, dont l'union nationale avait été rêvée par Amílcar Cabral et interrompue par le coup d'État de 1980, que Sarah Maldoror tourne Des Fusils pour Banta au début des années 1970, avant de réaliser deux films importants, Fogo, Île de feu (1977) et Carnaval à Bissau (1980). Après l'Angola, ces deux territoires, récemment indépendants, deviennent un autre front d'images dans l'œuvre de la cinéaste. Amílcar Cabral, cofondateur et secrétaire général du PAIGC, assassiné en 1973, était le parrain d'Annouchka de Andrade et le meilleur ami de Pinto de Andrade. Ils avaient étudié ensemble à Lisbonne, installés tous les deux à la Maison des étudiants de l'Empire, une résidence universitaire fondée en 1943 par le régime salazariste avec pour but la création d'une élite intellectuelle africaine mais qui fut surtout le berceau du nationalisme africain. Selon Pinto de Andrade, c'est à cette époque qu'ils commencèrent « à chercher comment redevenir [sic] des Africains ${ }^{8}$ ».

En 1951, Pinto de Andrade, Cabral et Neto, entre autres, fondent le Centre de recherche en études africaines. Deux ans plus tard, Pinto de Andrade crédité avec Francisco José Tenreiro coédite Caderno de Poesia Negra de Expressão Portuguesa, première anthologie de poésie africaine d'expression portugaise dont l'édition italienne sera préfacée par Pier Paolo Pasolini, suivie, en 1958, d'Antologia da Poesia Negra de Expressão Portuguesa. Ces deux publications sont fondamentales pour affirmer les formes culturelles africaines en tant que synthèse dialectique entre la tradition et la modernité. C'est en réalisant cette synthèse que la littérature anticoloniale constitue, selon Pinto de Andrade, une forme d'opposition et de résistance au système colonial et à ses normes littéraires ${ }^{9}$. Tel est le cas de l'œuvre de Luandino Vieira, référence majeure dans la filmographie de Sarah Maldoror, qui, inspirée des structures de la littérature orale africaine, renouvelle le portugais littéraire aux niveaux sémantique et syntactique pendant la lutte de libération angolaise.

\footnotetext{
${ }^{7}$ La date effective de fondation du MPLA reste controversée jusqu'à aujourd'hui selon les différentes sources.

8 Pinto de Andrade Mário. Préface à Cabral Amílcar (1980), Unité et lutte, Paris, Maspero, p. 13.

9 Pinto de Andrade Mário (1975), Antologia Temática de Poesia Africana. Cabo Verde, São Tomé e Príncipe, Guiné, Angola, Moçambique. I - Na Noite Grávida de Punhais, Lisbonne, Sá da Costa.
} 


\section{Image $n^{\circ} 5$ : Photo de tournage de Sambizanga (1973) au Congo-Brazzaville.}

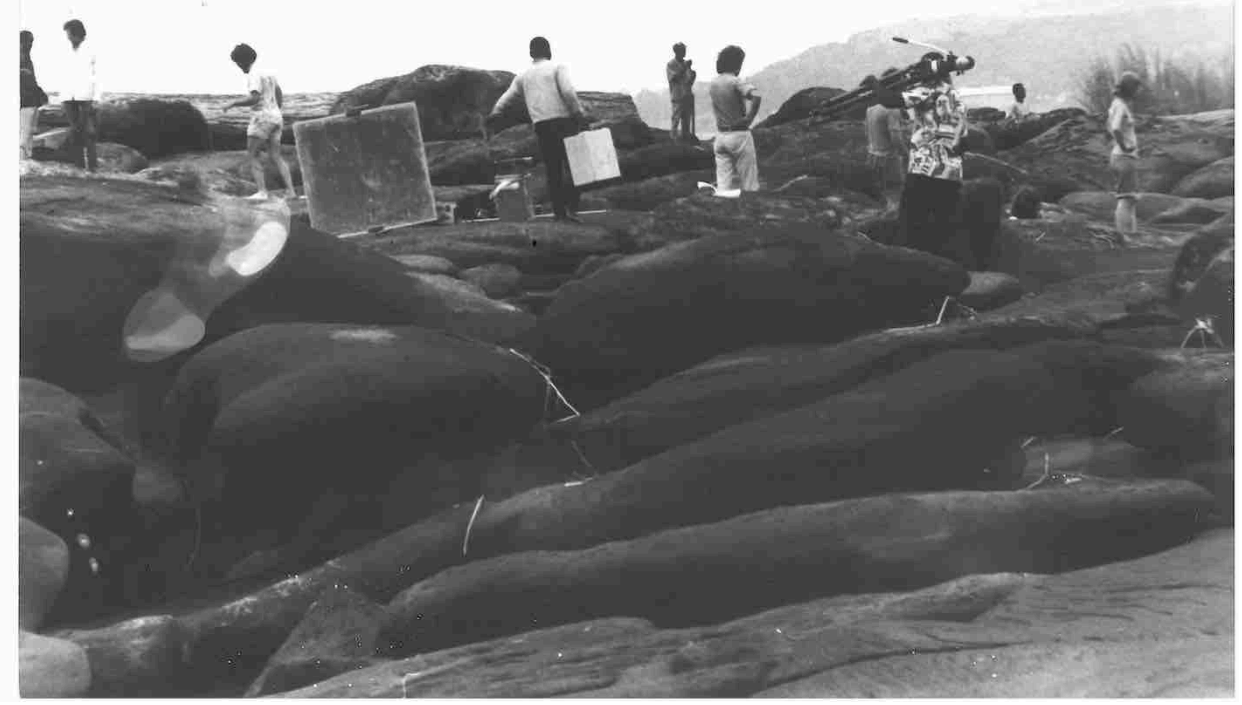

\section{De l'engagement et de l'invisibilité : Des fusils pour Banta (1971)}

L'œuvre de Sarah Maldoror s'inscrit dans la conjoncture du cinéma de Libération des années soixante et soixante-dix, un front esthétique et politique transnational et tricontinental (Amérique Latine, Afrique, Asie) définissant le cinéma comme un instrument de représentation mais aussi de transformation du monde. Le cinéma de Libération constitue l'une des « logiques culturelles ${ }^{10}$ » de ces mouvements dans lesquels on admet une continuité entre les formes artistiques et la lutte politique, l'avant-gardisme esthétique et l'avant-gardisme politique, et un lien conditionnel entre les révolutions nationales et leur extension spatiale - la révolution mondiale.

La longue et tortueuse histoire du long métrage Des fusils pour Banta (1971) témoigne de l'engagement de Sarah Maldoror dans les mouvements de libération, mais elle explique aussi l'invisibilité d'une part considérable du cinéma révolutionnaire de cette période. Les enjeux esthétiques et politiques de ces films, le contexte de leur production, leur situation d'énonciation ainsi que leur dimension ouvertement subjective, restent souvent mal compris des responsables des luttes de libération. L'histoire du film Des fusils pour Banta, considéré comme trop féministe et subjectif puisque centré sur un personnage féminin, fait ressortir les tensions entre émancipation et régulation à travers les processus de décolonisation et la mise en place d'États-nations africains. Les bobines auraient été confisquées à Sarah Maldoror lors de son retour à Alger, avant son expulsion du pays. Le film ne réapparaîtra jamais. Mais son histoire matérielle survit à travers son script et des photographies de tournage prises par Suzanne Lipinska, amie de Sarah Maldoror. Filmé par une femme noire à la tête d'une petite équipe technique de l'armée algérienne dans une zone libérée de la Guinée-Bissau en lutte

\footnotetext{
10 Jameson Fredric (1991), Postmodernism, or, The Cultural Logic of Late Capitalism, Durham, Duke University Press. Notre traduction.
} 
selon certaines sources ou bien au Congo-Brazzaville selon d'autres, Des fusils pour Banta affirme, en lien avec les propos de Julio García Espinosa dans son manifeste Pour un cinéma imparfait ${ }^{11}$, la puissance de la contingence technique et fait apparaître l'un des principes théoriques constitutifs du cinéma révolutionnaire de cette période: la collectivisation du cinéma et le passage de la représentation à des formes d'auto-représentation et de coreprésentation.

\section{Les carnavals de la victoire}

Image $n^{\circ} 6$ : Photogramme du générique de fin de Monamgambée (1969). La circulation des photographies prises par Augusta Conchiglia à la guerre de Libération de l'Angola

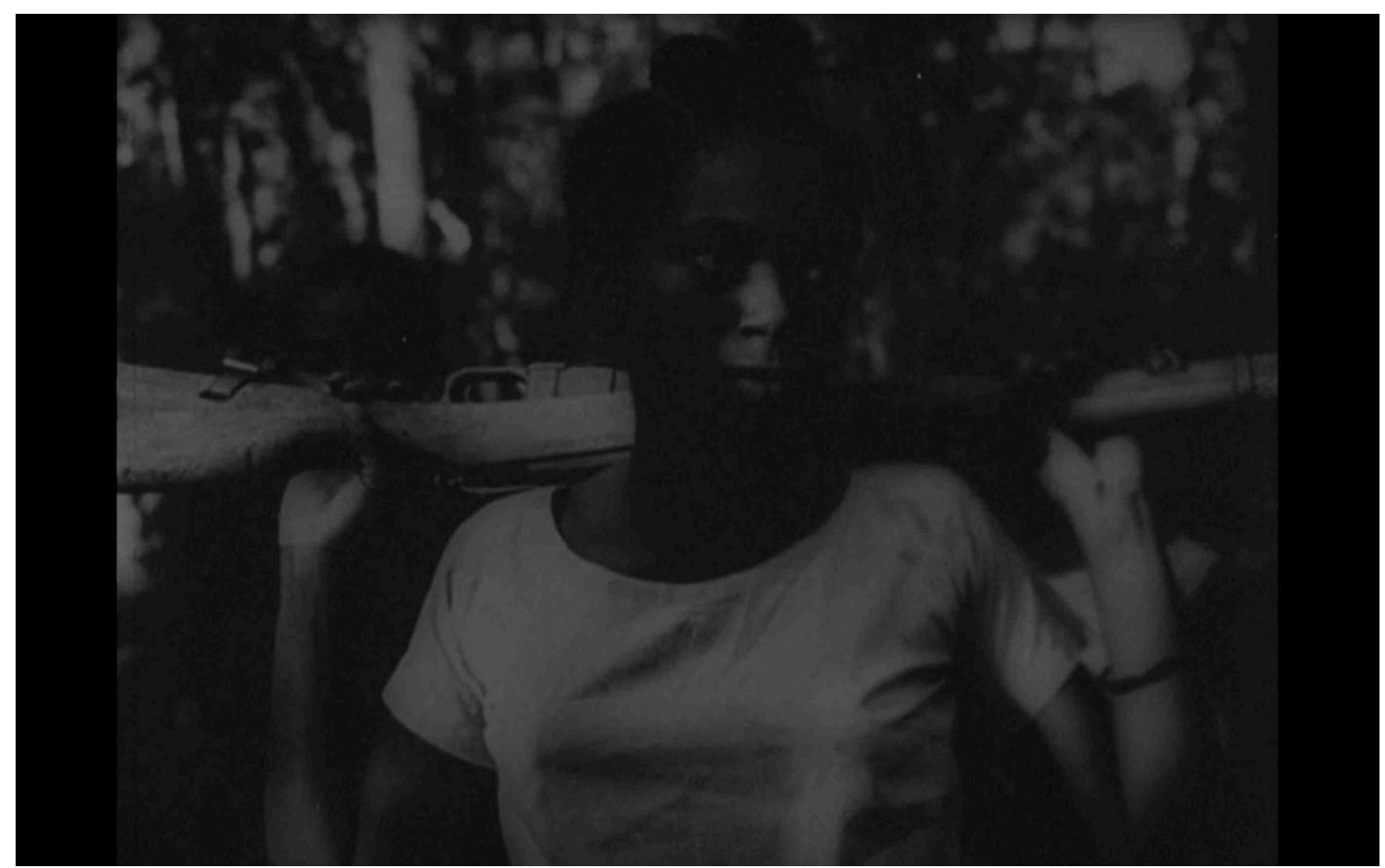

Le cinéma de Sarah Maldoror s'insère dans les débats idéologiques de son temps. Ses premiers films annoncent et formalisent "le réel à venir ${ }^{12}$ ", tandis que ceux réalisés après les indépendances figurent la complexe réalité postcoloniale. Dans Fogo, llle de feu, tourné à Fogo, au Cap-Vert, en 1977, la sensorialité devient une figure spatio-temporelle de l'expérience de I'insularité : fabulation, visions haptiques, regards-caméra, couleurs vibrantes, les arides paysages volcaniques comme toile de fond des corps féminins, anticipant certaines procédures formelles de Casa de Lava, de Pedro Costa, filmé à Fogo une quinzaine d'années plus tard ${ }^{13}$. Les logiques formelles de Carnaval à Bissau mettent en valeur la puissance du carnaval en tant que « pratique festive-révolutionnaire ${ }^{14}$ ». Robert Stam soutient que les traditions du carnaval, partagées par presque toutes les cultures de l'Atlantique noire, "signalent une vision

\footnotetext{
11 García Espinosa Julio (1979), « For an Imperfect Cinema », Jump Cut, 20, pp. 24-26.

12 Einstein Carl (2003 [1934]), Georges Braque, Bruxelles, Éditions La Part de I'CFil, 2003 (1934).

13 Deuxième long métrage de Pedro Costa, Casa de Lava porte sur les mémoires spectrales de la dictature fasciste et du colonialisme portugais, en même temps que sur les inter-identités (post)-coloniales avec en arrière-plan les géographies de Fogo.

14 Orenstein Claudia (1999), Festive Revolutions: The Politics of Popular Theater and the San Francisco Mime Troupe, Jackson, University of Mississippi, p. 29. Notre traduction.
} 
[cosmovision] alternative du monde des gens ordinaires ${ }^{15}$ » et donnent lieu «à des visions alternatives du monde [cosmovisions] ${ }^{16}{ }^{2}$. Suivant les conceptions de Bakhtine ${ }^{17}$, le théoricien nord-américain considère que « le carnaval embrasse une esthétique anti-canonique [sic] qui rejette I'harmonie formelle et l'unité en faveur de l'asymétrique, de l'hétérogène et du métissé ${ }^{18} »$. Robert Stam met en évidence, d'après Bakhtine et Henri Lefebvre ${ }^{19}$, la puissance subversive et révolutionnaire du carnaval. Or, le film de Sarah Maldoror, tourné à Bissau, témoigne, de la même manière que Carnaval da Vitória (Carnaval de la victoire, 1978), de I'artiste et cinéaste angolais António Olé, d'une alliance entre le carnaval et la révolution pendant les premières années d'indépendance. Ces masques dynamiques, dont les métamorphoses accompagnent les transformations survenues dans le pays entre la guerre de Libération et l'indépendance, reflètent le processus de formation de l'identité culturelle nationale. Les images tournées par Sana Na N'Hada pour Fogo, Île de feu et Carnaval à Bissau, qui auraient été remployées par Chris Marker20 dans Sans Soleil (1983), évoquent les intersections entre la politique et le rituel après l'indépendance. Mais elles sont aussi une marque visible des contradictions du projet de modernisation postcolonial, tout particulièrement du rôle du cinéma, envisagé comme un dispositif incarnant lui-même la modernité, dans la mise en place d'un projet politique qui se calque sur le modèle des Étatsnations européens et qui hérite des paradoxes de la modernité occidentale, notamment dans son rapport aux cultures extra-modernes considérées comme " primitives ».

Les films de Sarah Maldoror mettent aussi en évidence l'un des traits formels majeurs du cinéma de Libération : la circulation d'archives. Entre Fogo, Île de feu et Carnaval à Bissau et Sans Soleil, ou bien entre l'œuvre photographique d'Augusta Conchiglia et Monangambée, les images circulent. Dans le générique de fin de ce film, Maldoror remploie des photographies de la guerre de Libération en Angola prises par la photographe et cinéaste italienne qu'elle avait rencontrée au Festival Panafricain d'Alger de 1969. Ces connexions d'un médium à l'autre forment des nœuds formels, politiques et matériels, mais aussi des liens individuels et des affinités électives. Elles font aussi apparaître le principe de circulation qui oppose l'archive anticoloniale à la logique de domiciliation énoncée par Jacques Derrida ${ }^{21}$ à propos de l'archive. À travers ses circulations, l'archive anticoloniale se dissocie des configurations spatiales et temporelles qui définissent l'archive comme catégorie générale et l'archive coloniale en particulier et déjoue leurs rapports de savoir-pouvoir. Dans le même temps, cette dynamique situe le cinéma anticolonial, territoire du cinéma de Libération, au-delà de la catégorie stricte d'œuvre, et permet de le réinterpréter à partir d'un ensemble de pratiques relationnelles qui, s'opposant aux présupposés du modernisme occidental, s'élargissent aux processus de distribution, de réception et de resignification collective des images-sons cinématographiques.

\footnotetext{
15 Stam Robert, Porton Richard et Goldsmith Leo (2015). Keywords in Subversive Film/Media Aesthetics, Malden et Oxford, John Wiley \& Sons, p. 68. Notre traduction.

16 Ibid., p. 69. Notre traduction.

17 Bakhtine Mikhaïl (1970), L'ceuvre de François Rabelais et la culture populaire au Moyen Âge et sous la Renaissance, Paris, Gallimard, 1970.

18 Stam R., Porton R. et Goldsmith L., Keywords in..., op. cit., p. 69. Notre traduction.

19 Lefebvre Henri (1970), La Révolution urbaine, Paris, Gallimard.

20 En 1979, Chris Marker est venu à Bissau passer plusieurs mois avec les jeunes cinéastes guinéens Josefina Crato, Flora Gomes, José Bolama Cobumba et Sana Na N'Hada.

21 Derrida Jacques (1995), Mal d'archive, Paris, Galilée.
} 
Le coup d'État de novembre 1980 met fin au projet révolutionnaire de la Guinée-Bissau, ainsi qu'à son programme culturel. Compagne de Mario Pinto de Andrade, et amie de Luís Cabral, le frère d'Amílcar, Président du Conseil d'État entre 1973 et 1980, Sarah Maldoror ne peut rien pour sauver ses deux films guinéens. Le projet interrompu d'union nationale entre la Guinée-Bissau et le Cap-Vert figure comme une trace mémorielle dans Fogo, île de feu, qui devient du même coup un objet dissensuel et inclassable, invisible jusqu'à 2018.

\section{Le temps d'une larme ou d'un battement de paupières}

La filmographie de Sarah Maldoror fait trembler les limites thématiques et géographiques. Ses films traitent non seulement des luttes de libération africaines, mais aussi de la question de I'immigration en France (Un dessert pour Constance, 1980), de I'histoire politique de I'Amérique latine (Naissance de l'État, tourné au Panama en 1974, a récemment été retrouvé par Annouchka de Andrade), ou encore des grandes figures littéraires et artistiques auxquelles elle était liée: Aragon, Miró, le peintre russo-mexicain Vladimir Kibaltchitch ou encore la peintre colombienne Mercedes Hoyo. À Aimé Césaire, compagnon de toutes les luttes, à qui elle consacra plusieurs portraits filmés et dont elle adapta une pièce de théâtre, on pourrait laisser le dernier mot, comme une épitaphe à celle qui nous laisse sa colère et sa générosité en héritage : « II n'y aura pas de drapeau même noir pas de coup de canon pas de cérémonial. Ça sera très simple quelque chose qui de l'ordre évident ne déplacera rien mais qui fait que les coraux au fond de la mer les oiseaux au fond du ciel les étoiles au fond des yeux des femmes tressailliront le temps d'une larme ou d'un battement de paupières ${ }^{22}$ ».

Alice Leroy

LISAA/Université Gustave Eiffel (France)

Raquel Schefer

CEC/Université de Lisbonne (Portugal)

\section{Bibliographie}

BAKHTInE Mikhaïl (1970), L'ceuvre de François Rabelais et la culture populaire au Moyen Âge et sous la Renaissance, Paris, Gallimard, 1970.

CABRAL Amílcar (1980), Unité et lutte, Paris, Maspero.

CesaIRE Aimé (1956), Et les chiens se taisaient, Paris, Présence africaine. (2000 [1947]), Cahier d'un retour au pays natal, Paris, Présence africaine.

DeRRIDA Jacques (1995), Mal d'archive, Paris, Galilée.

EInstein Carl (2003 [1934]), Georges Braque, Bruxelles, Éditions La Part de l'CFil, 2003 (1934).

GARCía ESPINOSA Julio (1979), «For an Imperfect Cinema », Jump Cut, 20, pp. 24-26.

\footnotetext{
22 Césaire Aimé, Et les chiens se taisaient, Paris, Présence Africaine.
} 
JAMESON Fredric (1991), Postmodernism, or, The Cultural Logic of Late Capitalism, Durham, Duke University Press.

LefEBVRE Henri (1970), La Révolution urbaine, Paris, Gallimard.

MoURA Roberto (2002), "Le renouveau du dialogue cinématographique France-Brésil, et la contre représentation de la société brésilienne sous les gouvernements militaires dans le "cinéma invisible" des années 1969-90 », Histoire et sociétés de l'Amérique Latine, 2002, 14, pp. 11-32.

OREnSteIn Claudia (1999), Festive Revolutions: The Politics of Popular Theater and the San Francisco Mime Troupe, Jackson, University of Mississippi.

PINTo de Andrade Mário (1975), Antologia Temática de Poesia Africana. Cabo Verde, São Tomé e Príncipe, Guiné, Angola, Moçambique. I - Na Noite Grávida de Punhais, Lisbonne, Sá da Costa.

StAm Robert, Porton Richard et Goldsmith Leo (2015). Keywords in Subversive Film/Media Aesthetics, Malden et Oxford, John Wiley \& Sons. 